\title{
Recherche expérimentale et numérique sur le comportement d'une roue réversible au point de tracé et hors du point de tracé
}

\author{
Experimental and numerical investigation \\ of the behaviour of a reversible pump-turbine runner \\ at and off design conditions
}
G. Ventrone,
A. Mirandola,
G. Navarro,
Professeur
Professeur adjoint
Chercheur

«Istituto di Macchine» Université de Padoue (Italie)

\section{Introduction}

Depuis qu'Euler à le premier exprimé le couple moteur d'une turbo-machine en fonction de la modification du moment angulaire du fluide à l'entrée et à la sortie du rotor, la plupart des études théoriques ont porté davantage sur le comportement du rotor que sur l'interaction des divers éléments de la machine dans son ensemble.

Plusieurs critères ont été suggérés pour le calcul de la hauteur théorique mais ils ne correspondent pas au fonctionnement effectif de la machine, surtout dans des conditions hors conception. On peut espérer obtenir de meilleurs résultats à partir des développements récents dans les résolutions numériques de l'équation du mouvement d'un fluide dans une turbomachine.

Ces méthodes s'efforcent de décrire les configurations relatives de l'écoulement tout au long des canaux tournants ainsi qu'à la sortie du rotor. Le refoulement et le détachement de la veine fluide à l'intérieur de la roue mobile peuvent ainsi être prévus. On peut également calculer la déflexion relative de l'écoulement à partir du bord de fuite des aubes, dont on admet souvent qu'elle varie avec le débit, et par conséquent évaluer la hauteur théorique, c'èst-à-dire le travail spécifique $\mathrm{du}$ rotor.

En réalité, toutes les méthodes numériques que nous connaissons appliquent l'équation différentielle de l'écoulement relatif au rotor isolé, sans tenir compte de l'influence du corps ou de la partie fixe lorsqu'elles fixent les conditions aux frontières à l'entrée et à la sortie du rotor. Alors que cette méthode pourrait peutêtre être acceptable pour des rotors de turbines centripètes, elle est complètement aberrante pour des pompes et des compresseurs, où le réseau d'écoulement à la sortie du rotor varie considérablement avec le débit par suite de l'influence de la volute et/ ou du diffuseur.

Il est suggéré ici, en établissant les conditions aux frontières exigées pour résoudre l'équation différentielle de mouvement, d'introduire une fonction de distorsion dépendant de la géométrie de la partie fixe et du débit. Les résultats numériques pourraient être alors comparables aux valeurs de l'écoulement relevées par des essais sur une machine ordinaire.

La présente recherche a été effectuée sur un modèle d'étage de pompe-turbine réversible, muni d'un diffuseur de raccordement entre étages entièrement garni d'aubes. L'espace libre entre le rotor et le diffuseur à aubages a été entretoisé dans le fonctionnement en pompe, de façon à mettre en évidence la distorsion de l'écoulement produite par la partie fixe dans les conditions hors conception.

Les pales fixes ont alors été retirées, de façon à obtenir un tore où les conditions d'écoulement libre simulent à peu près celles qui existent en aval d'un rotor isolé. Les variations de vitesse et d'énergie avec le débit, telles qu'elles ont été détectées par les entretoises dans

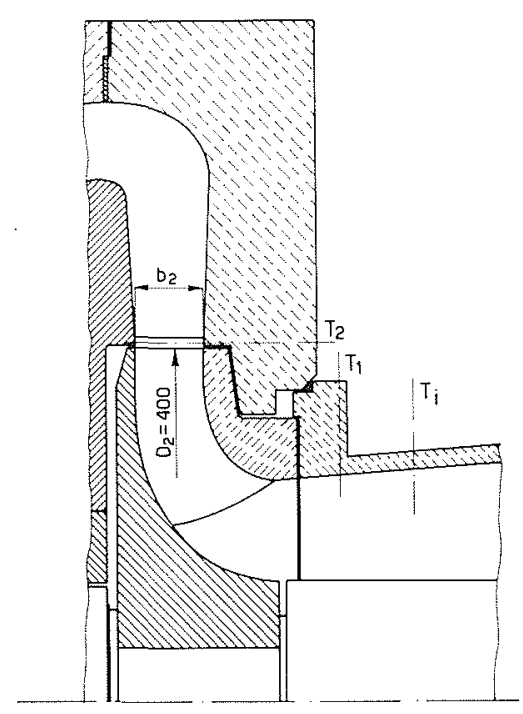

Figure 1 - Section méridienne du modèle d'étage de turbinepompe, qui a 7 aubes mobiles et 18 aubes de diffusion. Ti, T1, $\mathrm{T} 2$ : positions des sondes. 


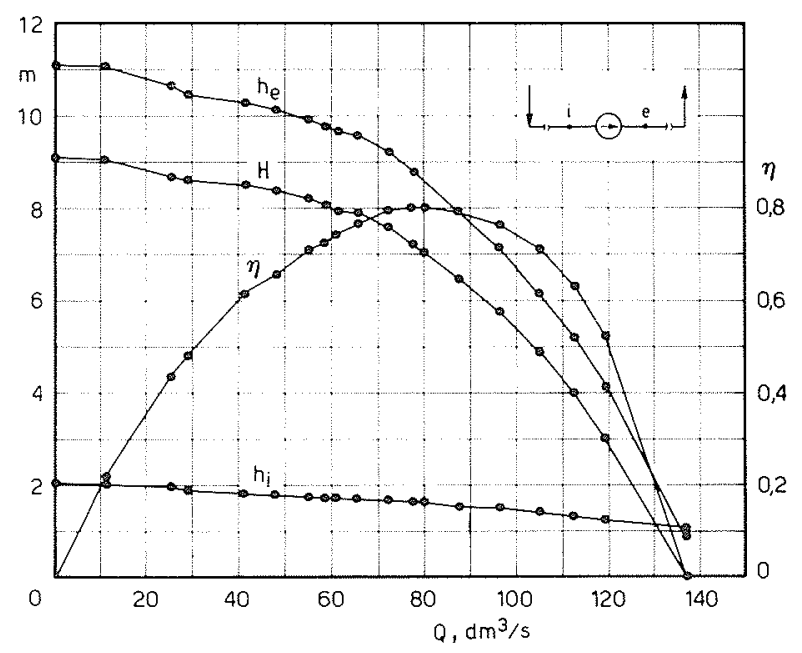

Figure 2 - Courbes caractéristiques en fonctionnement comme pompe à 600 tours/minute; $h_{i}, h_{e}$ : hauteurs de charge statiques dans l'entrée et la sortie de la machine.

l'écoulement, ont été finalement comparées avec les valeurs calculées. Le fonctionnement de l'étage en turbine a également été expérimenté, et a démontré que les conditions d'écoulement dans ce cas présentent moins de problèmes que dans le fonctionnement en pompe.

\section{Résultats expérimentaux}

Les recherches ont été effectuées sur un modèle d'étage intermédiaire d'une pompe-turbine réversible multi-étages, construit par Hydroart, SpA d'Italie. La figure 1 montre la coupe longitudinale de ce modèle. L'enveloppe extérieure ainsi que la couronne du rotor étaient en plexiglas, afin de permettre l'observation éventuelle des configurations de l'écoulement. Le rotor a 7 aubes dont l'angle du bord de fuite fait $27^{\circ}$. La figure 2 donne la hauteur totale d'élévation $\mathrm{H}$ produite dans le fonctionnement en pompe par l'étage avec diffuseur de raccordement à aubes et le rendement global en fonction du débit $Q$ à 600 tours/minute.

Une sonde cylindrique de $4,75 \mathrm{~mm}$ de diamètre extérieur à 5 trous fut utilisée pour entretoiser l'écoulement à l'emplacement $\mathrm{T} 2$ de la sortie du rotor. Les mesures furent comparées avec celles que l'on obtint avec une sonde à pointeau calibrée de $1,5 \mathrm{~mm}$, moins sensible aux effets de paroi, et dont l'influence sur l'écoulement est moindre. Des sondes furent également disposées aux points $\mathrm{Tl}$ et $\mathrm{Ti}$, du côté aspiration de l'étage.

Les valeurs moyennes pondérées de l'angle $\alpha_{2}$ de l'écoulement, de la composante tangentielle $c_{u 2}$ et de la composante méridienne $c_{m 2}$ de la vitesse absolue, mesurées au point $\mathrm{T} 2$, sont représentées sur la figure 3 .

$Q / A_{2}$ est le quotient du débit par la section d'écoulement à la sortie de la roue.

La courbe en trait plein $H_{t 2}-H_{t i}$ donne la différence entre les hauteurs totales de charge à la sortie du rotor (valeurs moyennes) et à l'entrée de l'étage modèle, ou le travail spécifique acquéri par le fluide; elle a

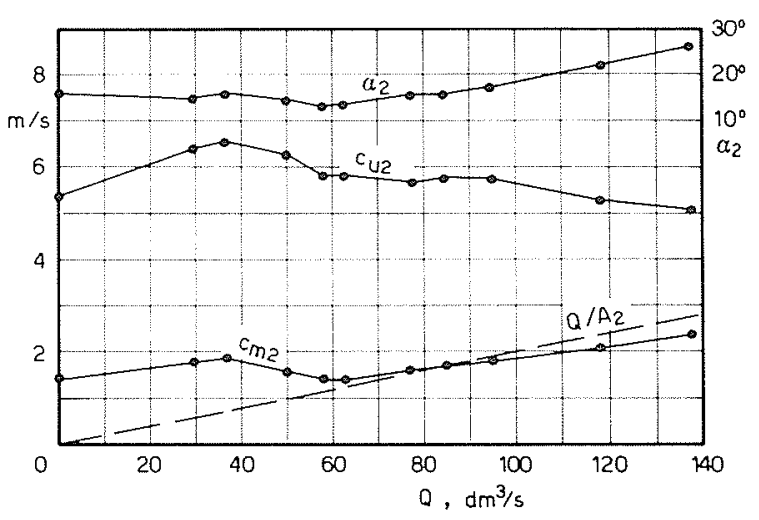

Figure 3 - Sondage à la sortie du rotor :

$\alpha_{2}=$ angle de l'écoulement,

$c_{u 2}$ et $c_{m 2}=$ composante tangentielle et méridienne de la vitesse,

$A_{2}=\pi D_{2} b_{2}$

presque la même allure que la hauteur totale d'élévation $\mathrm{H}$.

La relation $H_{t h}=\frac{u_{2} c_{u 2}}{g}$ donne le travail spécifique accompli par le rotor sur le fluide, $u_{2}$ étant la vitesse périphérique de la roue, si $c_{u 1}=0$.

En fait, quelques mouvements tourbillonnaires n'ont été détectés qu'à des débits voisins de zéro. Les considérations théoriques porteraient à s'attendre à ce que la courbe $H_{t h}$ soit différente de la courbe $H_{t 2}-H_{t i}$ seulement pour une partie proportionnelle aux pertes d'énergie dans le rotor, qui sont petites. Mais si l'on utilise les valeurs de $c_{u 2}$ tirées de la figure 3 pour calculer le travail spécifique $H_{t h}$, on obtient la courbe en pointillé de la figure 4 , qui diffère considérablement de la courbe $H_{t 2}-H_{t i}$, qu'elle coupe pour $Q=$ $85 \mathrm{dm}^{3} / \mathrm{s}$. C'est le débit pour lequel $c_{m 2}$ et $Q / A_{2}$ coïncident dans la figure 3 .

On peut montrer que, pour $Q=85 \mathrm{dm}^{3} / \mathrm{s}$, les pertes dues à l'incidence à l'entrée des aubes du diffuseur étaient presque nulles [1]. En conséquence, pour des débits inférieurs ou supérieurs, l'écoulement dans le tore apparait progressivement déformé par l'action réciproque du rotor et des aubes fixes.

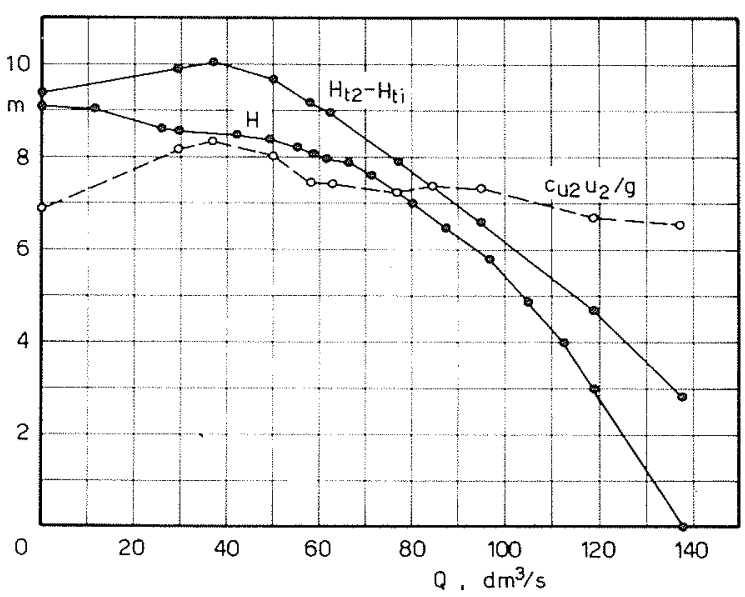

Figure 4 - Augmentation de la hauteur totale du fluide à travers le rotor relevé par le sondage, comparé avec la hauteur totale d'élévation et la hauteur théorique (ligne pointillée). 
La courbe $H_{t 2}-H_{t i}$ de la figure 4 perd pour $Q=$ $58 \mathrm{dm}^{3} / \mathrm{s}$ l'allure quasi-rectiligne qu'elle avait dans la région des débits élevés, et s'infléchit encore pour $Q=36 \mathrm{dm}^{3} / \mathrm{s}$. Le même comportement apparait sur la caractéristique de la hauteur totale d'élévation $\mathrm{H}$ de la figure 2. Ce sont des signes évidents que des décollements de filets et des inversions de l'écoulement se sont produits dans les canaux du rotor.

En vue de vérifier ce qui précède, un flux d'air fut injecté au moyen d'une sonde à pointeau située à l'entrée du rotor (point $T 1$ de la figure 1). Le cheminement du flux d'air dans un canal en rotation fut observé visuellement au moyen d'un stroboscope. Aucune particularité ne put être détectée dans l'écoulement pour des débits supérieurs à $60 \mathrm{dm}^{3} / \mathrm{s}$. Vers $Q=50 \mathrm{dm}^{3} / \mathrm{s}$, toutefois, on vit l'écoulement décoller de la paroi en dépression de l'aube, et vers $30 \mathrm{dm}^{3} / \mathrm{s}$, il parut circuler à nouveau à l'intérieur du passage près de la paroi en compression de l'aube. Le refoulement remonta légèrement au-dessus du point $T 1$, avec un mouvement tourbillonaire qui croissait quand le débit tendait vers zéro.

L'étape suivante des recherches consista alors à examiner comment se comporterait effectivement le rotor s'il n'était pas soumis à l'influence de son environnement, de façon à pouvoir comparer plus valablement les résultats expérimentaux à ceux des méthodes numériques.

Etant donné que le diffuseur de raccordement de l'ensemble expérimental de la figure 1 était caractérisé par un taux radial élevé, nous avons procédé au démon-
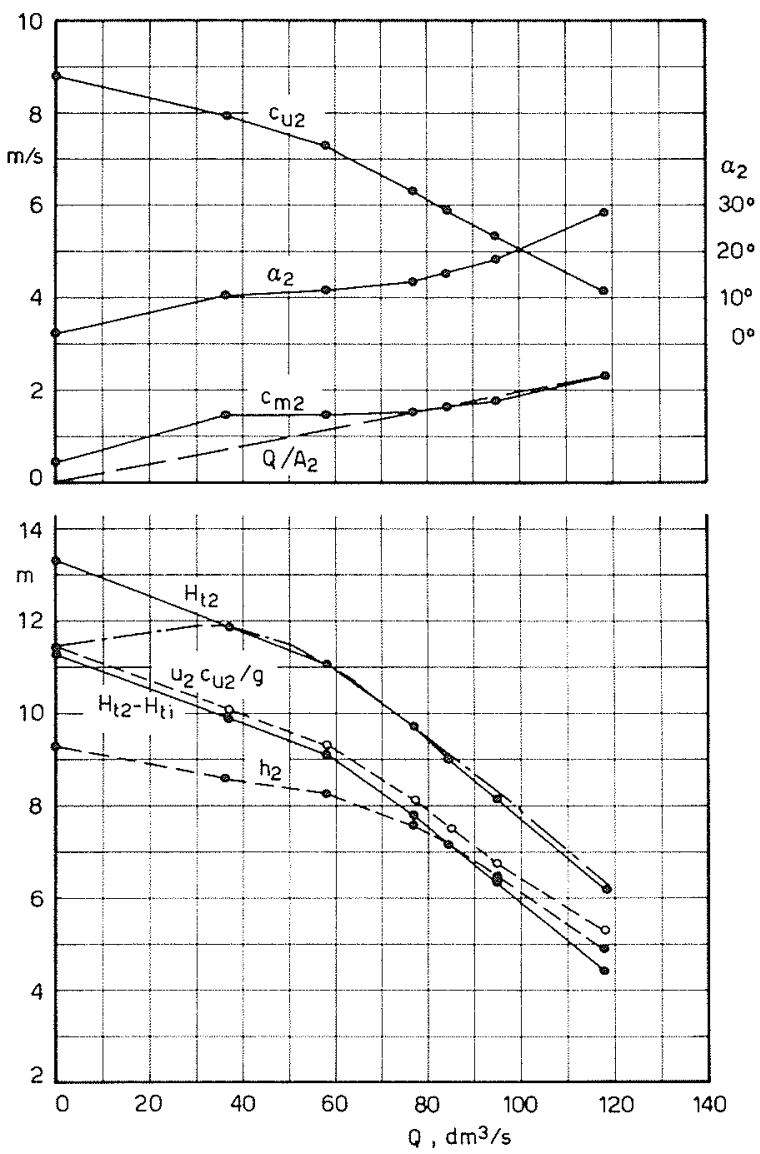

Figure 5 - Sondage à la sortie du rotor avec diffuseur sans aubes. tage des aubes du conduit fixe, afin d'obtenir un tore sans obstacle, où seraient réalisées des conditions d'écoulement libre comparables aux configurations d'écoulement à l'extérieur d'un rotor isolé.

On ne peut évidemment pas s'attendre à ce que la similarité s'étende sur toute la gamme des débits, car le tore se comporte comme un diffuseur radial sans aubes, de sorte qu'il y a lieu de supposer une influence sur le rotor, en particulier aux faibles débits.

La figure 5 montre les valeurs moyennes pondérées des paramètres de l'écoulement obtenues en entretoisant le tore libre à la périphérie du rotor.

Les mesures présentent une meilleure concordance avec les prévisions théoriques. La courbe $\left(u_{2} \cdot c_{u 2} / g\right)$, calculée à partir des lectures à la sonde des vitesses tangentielles, est maintenant, pour toute la gamme des débits, plus haute que la courbe $H_{t 2}-H_{t i}$, cause les pertes dans la roue. La courbe mixtiligne montre l'allure de $H_{t 2}$ relevée dans le cas précédent. On voit qu'elle coincide avec la nouvelle courbe $H_{t 2}$, sauf pour les débits inférieurs à $38 \mathrm{dm}^{3} / \mathrm{s}$, pour lesquels les pertes par brassage et recirculation, amplifiées par le diffuseur à aubes, commencent à devenir non négligeables. Dans la gamme des débits compris entre zéro et $38 \mathrm{dm}^{3} / \mathrm{s}$, une certaine discordance est encore apparente dans la courbe de $c_{m 2}$; elle est cependant bien inférieure à celle du cas précédent (Cf. Fig. 3). Il est intéressant de noter que les pressions totales lues à la sonde ne sont pas très affectées par l'ensemble fixe sauf aux très faibles débits, pour lesquels les pertes d'énergie dues aux interférences et au brassage sont prépondérantes.

La courbe $u_{2} c_{u 2} / g$ de la figure 5 a la même allure que la courbe prévue par la théorie, c'est-à-dire que la hauteur théorique croît quand le débit décroit.

La courbe $H_{t h}$ peut être calculée en évaluant la déflexion de l'écoulement à la sortie de la roue, définie comme la différence entre la composante d'Euler et la composante réelle $c_{u 2}$; on peut utiliser, par exemple, la méthode de Busemann.

Les courbes en pointillé de la figure 6 ont été obtenues par cette méthode ; la déflexion relative a été gardée constante, sauf par rapport à la variation des fuites et du blocage suivant le débit.

On voit que ces courbes coïncident assez bien avec les courbes expérimentales (en traits pleins) reproduites d'après la figure 5 .

La puissance absorbée par la machine à diffuseur sans aubes est résultée inférieure à celle du cas précédent.

La puissance au frein pour un débit nul ne représente dans ce cas que $37 \%$ de la puissance utile contre $58 \%$ dans le cas précédent.

$\mathrm{Si}$, pour un débit nul, on tient compte des pertes dues au frottement mécanique, aux jeux et aux fuites, et que l'on porte dans l'équation de puissance nette la hauteur d'Euler à débit nul, il en résulte une valeur invraisemblablement trop faible du taux de l'écoulement de recirculation. On peut ainsi en déduire que la hauteur théorique à débit nul est peut-être inférieure à la hauteur d'Euler à débit nul. La valeur réelle doit probablement approcher de la valeur correspondante de la figure 6. 


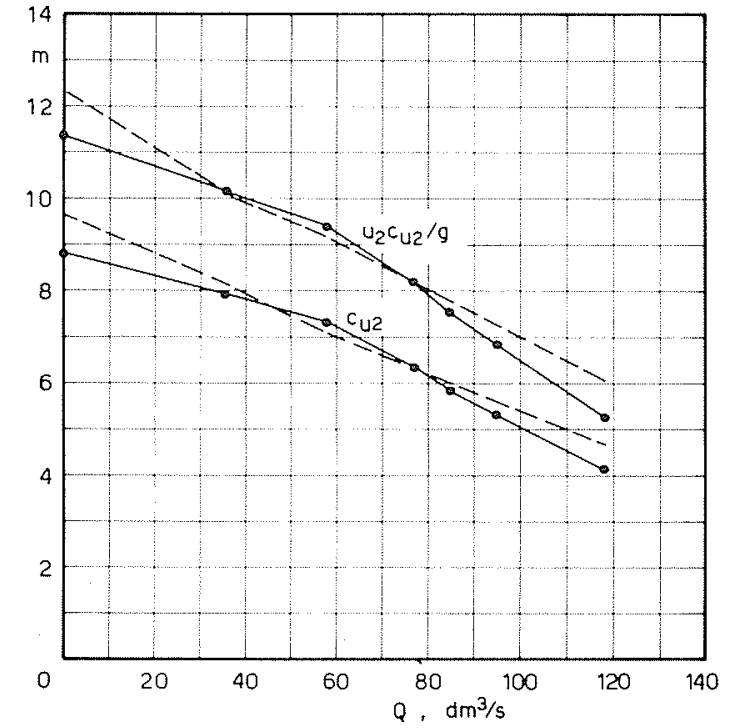

Figure 6 - Valeurs mesurées (traits pleins) et calculées (lignes pointillées) des vitesses tangentielles et de la hauteur théorique du rotor, en absence des aubes de diffusion.

Dans la discussion des graphiques des figures 3 et 4 , il a été souligné que, pour $Q=85 \mathrm{dm}^{3} / \mathrm{s}$, les valeurs mesurées de la vitesse de l'écoulement et de la pression totale paraissaient en accord avec l'équation de continuité et la hauteur théorique. Les discordances constatées aux débits inférieurs et supérieurs ont été attribuées à l'interaction du rotor et des aubes du diffuseur, qui perturbaient le parcours libre des filets dans le tore. La situation a été illustrée par le schéma de la figure 7 , où la droite pleine représente la direction de l'écoulement non perturbé telle qu'elle résulte d'une adaptation correcte des angles du rotor et du diffuseur. Pour des valeurs plus petites ou plus grandes, les filets quittant le rotor changent de direction conformément aux prévisions théoriques, mais sont forcés de pénétrer dans le conduit suivant l'inclinaison $\alpha_{d}$ des aubes fixes. Par conséquent, la sonde placée dans l'intervalle annulaire détectera un angle d'écoulement qui dépend étroitement de la direction presque sans changement de l'écoulement dans les aubes fixes. La pression totale, par contre, est très peu influencée par la partie fixe, sauf aux faibles débits, où les pertes par brassage et la recirculation sont prépondérantes.

Dans les pompes à volute, la configuration de l'écoulement autour de la périphérie extérieure de la roue mobile est beaucoup plus compliquée, parce qu'aux conditions autres que le point de concordance rotorvolute, la vitesse et la pression varient considérable-

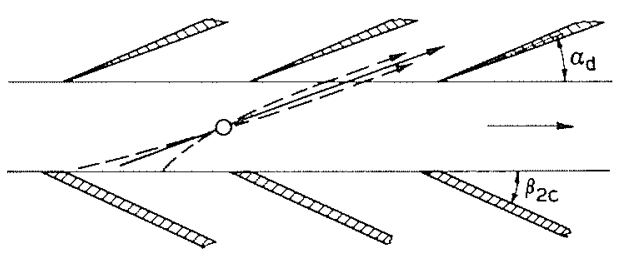

Figure 7 - Représentation schématique de l'effet de distorsion des aubes fixes sur le courant à la sortie du rotor.
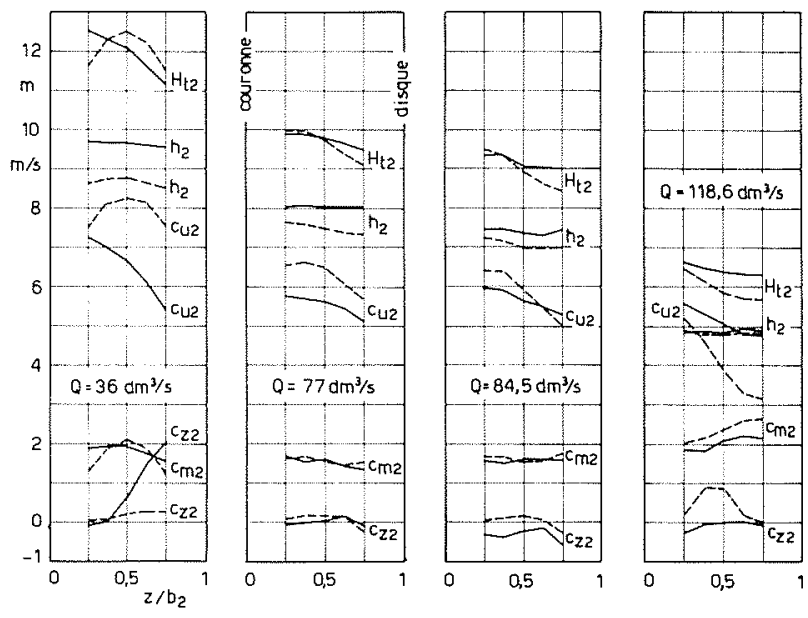

Figure 8 - Influence des aubes fixes sur la distribution axiale des vitesses et des pressions (- avec aubes, - - sans aubes) ; $c_{z 2}=$ composante axiale de la vitesse (sens positif de la couronne vers le disque).

ment avec la distance à partir du bec. La figure 8 montre que même la distribution axiale des paramètres d'écoulement dans la section T2 est influencée par les aubes fixes.

Les aubes fixes furent alors replacées dans le conduit fixe, et la machine essayée en turbine. Les courbes caractéristiques sont données par la figure 9 .

La figure 10 représente les paramètres de l'écoulement relevés en entretoisant l'espace libre autour du rotor. Comme il a été dit dans l'introduction, les courbes de pression et de vitesse ne sont pas en désaccord avec les prévisions théoriques, et peuvent être établies sans difficulté sérieuse, au moins pour la gamme la plus utile de fonctionnement de la turbine.

La hauteur théorique (ligne pointillée de la figure 9), qui s'exprime maintenant par $g H_{t h}=\left(u_{2} c_{u 2}-u_{1} c_{u 1}\right)$ a été calculée en tenant compte de la déviation moyenne de l'écoulement au bord interne des aubes du rotor, suivant la même méthode que nous avions adoptée pour lis rotor de pompe.

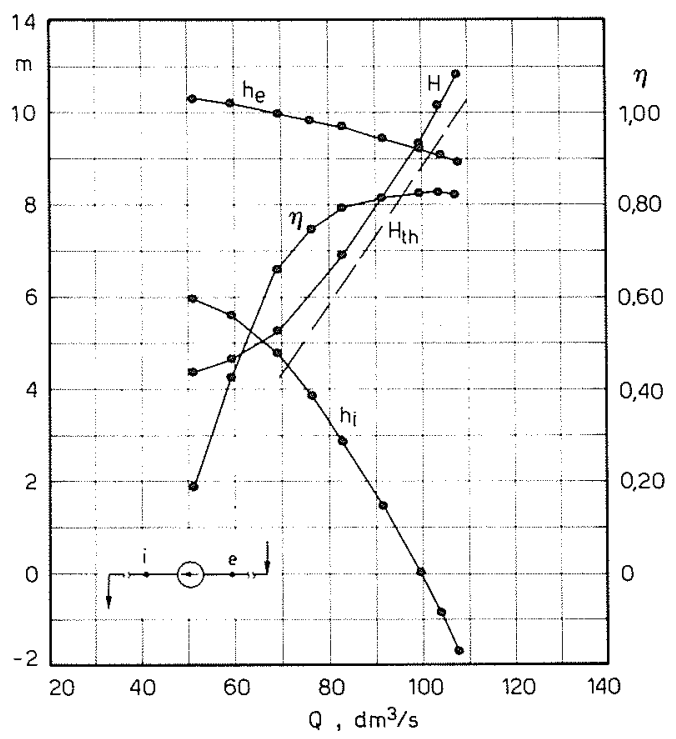

Figure 9 - Courbes caractéristiques en turbinage à 600 tours/ minute. 


\section{Considérations sur les méthodes numériques}

L'analyse numérique des configurations d'écoulement dans une turbomachine repose sur la résolution d'équations différentielles aux dérivées partielles définies par Wu [2] et Stanitz [3] : des exemples d'une application simplifiée ont également été suggérés par Stanitz et Katsanis [4], [5], [7].

La présente discussion a pour principal objet l'analyse de l'écoulement, considéré comme incompressible, sur des surfaces de révolution entre deux aubes.

La disposition des filets sur la surface méridienne est supposée connue.

L'équation différentielle aux dérivées partielles de l'écoulement entre les aubes est alors:

$\frac{1}{r}\left[\frac{\partial w_{m}}{\partial \vartheta}-\frac{\partial\left(r \cdot w_{u}\right)}{\partial m}\right]=\frac{w_{n}}{w_{u}} \cdot \Omega_{w_{u}}+2 \cdot|\vec{\omega}| \cdot \operatorname{sen} \gamma ;(1)$

$w$ est la vitesse relative, avec les indices $m$ et $u$ pour les composantes méridiennes et tangentielles, $\Omega_{w}$ la vorticité relative, $\omega$ la vitesse angulaire, $\theta$ la coordonnée angulaire, $\gamma$ la pente de la tangente au filet méridien relatif à l'axe du rotor.

En introduisant la fonction du courant $\psi \%$ et en transformant les coordonnées intrinsèques $(\partial-9)$ en un système de coordonnées curvilinéaires orthogonales $(\gamma-\theta)$, comme suggéré par ailleurs par l'un des auteurs [6], l'équation (1) devient :

$$
\begin{aligned}
& \frac{\partial}{\partial \vartheta}\left[\frac{R_{c}}{b \cdot r}\left(\frac{\partial \Psi \%}{\partial \vartheta}\right) \frac{Q}{100 \cdot n_{p}}\right] \\
& +\frac{\partial}{\partial \gamma}\left[\frac{r}{b \cdot R_{c}}\left(\frac{\partial \psi \%}{\partial \gamma}\right) \frac{Q}{100 \cdot n_{p}}\right] \\
& =r \cdot R_{c} \cdot\left[2 \cdot \omega \cdot K_{E} \cdot \operatorname{sen} \gamma+\frac{w_{n}}{w_{u}} \cdot \Omega_{w_{u}}\right]+D
\end{aligned}
$$

où

$$
D=\frac{w_{m}}{k_{m}} \cdot \frac{\partial R_{c}}{\partial \theta},
$$

$k_{E}=+1$ pour la marche en pompe et -1 pour la marche en turbine, et $R_{c}$ est le rayon de courbure du filet méridien (le sens positif est dans la direction négative des coordonnées normales $n$ ).

En utilisant la méthode de résolution par éléments finis, les expressions de l'équation fonctionnelle $F$ et de la minimisation $\left(\partial \chi^{e} / \partial \psi_{J} \%\right)$ de la fonction variationnelle $\chi^{(e)}$ sont les suivantes :

$$
F=\frac{1}{2 \cdot b} \cdot\left[\frac{R_{c}}{r} \cdot\left(\frac{\partial \psi \%}{\partial \vartheta}\right)^{2}+\frac{r}{R_{c}} \cdot\left(\frac{\partial \psi \%}{\partial \gamma}\right)^{2}\right] \cdot \frac{Q}{100 \cdot n_{p}}-\left\{r \cdot R_{c} \cdot\left[2 \cdot \omega \cdot K_{E} \cdot \operatorname{sen} \gamma+\frac{w_{n}}{w_{u}} \cdot \Omega_{w_{u}}\right]+D\right\} \cdot \Psi \% ;
$$

$$
\begin{aligned}
\frac{\partial \chi^{(e)}}{\partial \Psi_{J} \%}= & \frac{Q}{100 \cdot n_{p}} \cdot \iint_{s}(e)\left\{\frac{R_{c}}{b \cdot r} \cdot\left[\sum_{i=1}^{n t} \psi_{i} \% \cdot \frac{\partial N_{i}}{\partial \vartheta}\right] \cdot \frac{\partial N_{J}}{\partial \vartheta}+\frac{r}{b \cdot R_{c}} \cdot\left[\sum_{i=1}^{n t} \psi_{i} \% \cdot \frac{\partial N_{i}}{\partial \gamma}\right] \cdot \frac{\partial N_{J}}{\partial \gamma}\right\} \cdot d \vartheta \cdot d \gamma+ \\
& \iint_{s}(e)\left\{r \cdot R_{c} \cdot\left[2 \cdot \omega \cdot K_{E} \cdot \operatorname{sen} \gamma+\frac{w_{n}}{w_{u}} \cdot \Omega_{w_{u}}\right]+D\right\} \cdot N_{J} \cdot d \vartheta \cdot d \gamma+\oint_{c}(e) \mathrm{q} \cdot \mathrm{N}_{J} \cdot d_{s}=0
\end{aligned}
$$


Les conditions aux frontières pour la totalité de la frontière $A B C D E F G H$ sont exigées :

1) le long de $\mathrm{HG}, \psi \%=0$

2) le long de $\mathrm{CD}, \psi \%=100$

3) le long de $\mathrm{AH}$ et de $\mathrm{BC}$, il existe des conditions périodiques, qui sont :

$$
\begin{aligned}
(\Psi \%)_{B C} & =(\Psi \%)_{A H}+100 ; \\
\text { et }(\partial \Psi \% / \partial t)_{B C} & =(\partial \Psi \% / \partial t)_{A H}
\end{aligned}
$$

où $t$ est la normale à la frontière

4) le long de $D E$ et de $G F$, il existe des conditions périodiques, qui sont :

$$
\begin{aligned}
(\Psi \%)_{D E} & =(\Psi \%)_{G F}+100 ; \\
\text { et }(\partial \Psi \% / \partial t)_{D E} & =(\partial \Psi \% / \partial t)_{G F}
\end{aligned}
$$

Dans l'établissement des conditions aux frontières le long de $F E$, Katsanis utilise une valeur uniforme de $w_{u}$ qui doit être obtenue à partir d'une expérience précédente, et qui reste constante au cours des itérations [7].

Cependant des situations du plus haut intérêt pratique sont celles où la distribution du vecteur vitesse relative à la limite externe du rotor est inconnue. Dans de tels cas, il peut être très difficile d'arriver à une solution du problème.

En variante, l'un des auteurs a suggéré de dériver la valeur moyenne de la composante $w_{u}$ à la limite externe du domaine fluide en intégrant le long de $F E$. (fig. 11) l'expression suivante, qui suppose que l'énergie totale est conservée entre $G D$ et $F E$ :

$$
\begin{array}{r}
\left(w_{u}\right)_{F E}=\frac{r_{G D}}{100 \cdot r_{F E}} \cdot \int_{\psi_{G} \%}^{\psi_{D}^{\%}}\left(w_{u} \cdot d \psi \%\right)_{G D} \\
-\frac{\omega}{r_{F E}} \cdot\left(r_{F E}^{2}-r_{G D}^{2}\right)
\end{array}
$$

Les méthodes précédentes peuvent donner des résultats satisfaisants à la condition qu'aucune action extérieure ne soit exercée sur l'écoulement dans le rotor, autre que les forces de pression échangées par le rotor lui-même.

Dans les pompes et les compresseurs, cette exigence ne peut être satisfaite que pour le débit auquel les conditions d'écoulement à la sortie du rotor correspondent parfaitement à celles de la parti fixe adjacente.
Dans une turbine centripète, au contraire, les conditions d'écoulement à l'entrée du rotor sont connues, ou peuvent être déterminées avec peu de difficulté, et à la sortie, l'on peut admettre qu'elles soient peu influencées par le corps.

Dans les rotors de pompes à volute ou à diffuseur garni d'aubes, les conditions aux frontières à la sortie du rotor devraient être exprimées par une équation où il faut tenir compte des caractéristiques géome triques du corps, du débit et des gradients circonférentiels de vitesse relative et de pression. Ces gradients expriment les effets perturbateurs du corps sur l'écoulement (Cf. fig. 7). De plus, dans les pompes à volute, les conditions à la frontière à la sortie d'un conduit tournant changent avec la distance à partir du bec de la volute, et par conséquent dépendent du temps.

La fonction de distorsion $T$ peut s'exprimer comme suit sous forme implicite

$$
T=f\left(\lambda, \varphi, \frac{\partial p}{r . \partial \vartheta}, \frac{\partial w}{r . \partial \vartheta}, \tau\right) ;
$$

$\lambda$ traduit les propriétés géométriques du corps, $\varphi$ est le nombre de l'écoulement et $\tau$ indique la dépendance du temps de la fonction $T$.

\section{Bibliographie}

[1] VENTRONE G., MIRANDOLA A. - Analisi sperimentale di un diffusore-convogliatore palettato di una reversibile pluri-stadio per impianto di accumulazione idrica. Tecnica Italiana, n. 1, 1981.

[2] WU C. - A General Theory of Three-Dimensional Flow in Subsonic and Supersonic Turbomachines of Axial, Radial, and Mixed-Flow Types, Trans. ASME, vol. 74, 1952, pp. $1363-1380$.

[3] STANITZ J.D. - Some Theoretical Aérodynamic Investigations of Impellers in Radial and Mixed Flow Centrifugal compressors - Trans, ASME - Vol. 74 - 1952 - pp. 473-497.

[4] KATSANIS T. - Use of Arbitrary Quasi-Orthogonals for Calculating Flow Distribution in the Meridional Plane of a Turbomachine, NASA TN D-2546, 1964.

[5] KATSANIS T. - Computer Program for Calculating Velo. cities and Streamlines on a Blade-to-Blade Stream Surface of a Turbomachine NASA TN D-4525, 1968.

[6] NAVARRO G. - Formulazioni di sistemi di Coordinate per Superfici "Blade-to-Blade" di una Turbomacchina, L'Aerotecnica Missili e Spazio, vol. 60, n. 2, 1981, pp. 106-116.

[7] KATSANIS T. - Fortran Program for Calculating Transonic Velocities on a Blade-to-Blade Stream Surface of a Turbomachine. NASA TN D - 5427, 1969. 


\section{Discussion}

Président : R. BALLAY

Le Président remercie $M$. VENTRONE de sa communication et ouvre la discussion.

M. GUITON. - J'ai été très intéressé par la comparaison des essais avec aubes et sans aubes dans le diffuseur, essais qui donnent des puissances différentes, et particulièrement dans les petits débits, et par les différences des valeurs de $c_{u 2}$ que cela donnait avec aubes et sans aubes.

J'au rais voulu savoir quelle était la valeur de l'entrefer relatif.

Le Président. - D'après ce que MM. VENTRONE et BORCIANI viennent de préciser l'entrefer relatif $\frac{D_{3}-D_{2}}{D_{2}}$ est de

M. BORCIANI. - Actuellement les groupes multiétages reversibles de l'usine de Chiotas, dont 3 étudiés par HYDROART, sont en service. Il est intéressant de savoir qu'il y a une bonne correspondance entre la formule de transposition appliquée au rendement pour le modèle HYDROART, le rendement mesuré par l'ENEL avec la méthode thermodynamique.

M. CERA VOLA. - Je dois préciser que le modèle d'un des étages de la pompe-turbine multiétage qui a été utilisé pour les essais dans le laboratoire de l'Université de PADOUE n'a pas été copié industriellement. Cela est dû au fait que le stator a une dimension radiale très élevée, surtout si l'on tient compte du grand nombre d'aubes (18). Dans les applications industrielles de multiétages reversibles, HYDROART a adopté des diffuseursconvoyeurs à 16 ou 11 ailettes.

M. VENTRONE. - Le nombre des aubes du diffuscur ne peut pas influencer qualitativement les résultats des recherches, mais seulement les faire varier du point de vue quantitatif.

M. le Président. - Que pensez-vous de la réponse d'une sonde à 5 trous à la sortie d'une roue, à une quelconque distance de la roue!

M. VENTRONE. - La sonde donne la valeur moyenne dans le temps. Il y a une première erreur quand elle fonctionne dans cette situation : elle tend à relever la valeur de la pression totale à cause des sillages. La deuxième cause d'erreur est due au fait que lorsque l'on introduit la sonde, les lignes de courant sont déviées entre l'extrémité de la sonde et la paroi lorsque l'on se rapproche trop de cette dernière.

De ce fait, on a donc utilisé deux sondes : lors de l'expérience, une sonde à 5 trous et une autre sonde de $1,5 \mathrm{~mm}$ construite dans le laboratoire de PADOUE.

La sonde ne donnait pas l'angle du flux mais était extrêmement utile pour comparer les indications obtenues avec celles de la sonde à 5 trous.

La sonde à 5 trous ayant un diamètre de $4,75 \mathrm{~mm}$ on avait des doutes sur la possibilité de distorsion de flux provoquée par cette sonde.

\section{Abstract \\ Experimental and numerical investigation of the behaviour of a reversible pump-turbine runner at and off design conditions}

Object of this paper is the comparison of actual flow configurations in a turbomachine with flow configurations estimated by numerical procedures.

Blade-to-blade solutions of the differential equation of motion are generally referred to an isolated runner, and in fixing the boundary conditions at the impeller periphery the influence of the casing or of the stationary system is completely disregarded. While this may be acceptable for centripetal turbine runners, it gives unsatisfactory results in pumps and compressors, where flow pattern at the runner outlet changes considerably with the discharge rate because of the influence of the volute and/or the diffuser.

The present investigation has been conducted on a model reversible pump-turbine stage fitted with a fully bladed diffuserconveyor. The runner tip clearance has been traversed in pump operation in order to examine the distorsion of the flow induced by the stationary system. The averaged values of flow angle, velocity and total pressure better agreed with theoretical estimates only at the matching point of runner and diffuser, which occurred at a discharge different from the b.e.p. rate. At lower or higher rates the averaged value of the meridional component of the absolute velocity differed considerably from actual through-flow. And also, the runner input head obtained from the averaged values of the tangential component of the velocity, which on account of the impeller losses was expected to be always greater than the total head rise through the runner, appeared to be smaller than the latter at flows below the design rate and greater at higher flows. The discrepancies have been ascribed to the interaction of runner and stationary vanes, which distorted the flowlines in the clearance annulus. It is also shown that fewer interference problems are present in turbine operation.

The stationary vanes were then removed from the diffuser channel so as to obtain an unobstructed annulus where free flow conditions would set up comparable to those existing outside an isolated runner. Flow measurements showed now better consistency with theoretical estimates. It could also be appreciated that the ratio of slip velocity to runner tangential velocity did not change appreciably with the rate of flow. Axial distribution of velocity and pressure in the clearance annulus was also considerably affected by the presence of the diffuser vanes.

Known numerical solutions of the equation of motion may yield satisfactory results provided no external action is exerted on the runner flow other than the pressure forces exchanged by the runner itself. For pump runner with volute or vaned diffuser casing, the boundary conditions at the impeller exit should be defined by an equation expressing the distorting effects of the stationary system on the flow. 